\title{
Assessment of trends in socioeconomic inequalities in cancer screening services in Korea, 1998-2012
}

Sujin $\mathrm{Kim}^{1}$ and Jongnam Hwang ${ }^{2^{*}}$

\begin{abstract}
Background: This study aimed to examine how income-related inequalities in screening services for gastric and colorectal cancer in Korea have changed over the past decades, along with the implementation of the national cancer screening program, and also to quantify each contribution from various socio-demographic factors income-related inequalities with respect to these cancer screening services.

Methods: Three cycles (1998, 2005, and 2010-2012) of Korea National Health and Nutrition Examination Survey (KNHANES) were utilized. To measure income-related inequalities in the use of gastric and colorectal cancer, individuals over the age of 40 and the age of 50 were included respectively, and the Concentration Index (Cl) was calculated for each cycle. To identify and quantify contribution from each socio-demographic factor, decomposition of the Cls was conducted.

Results: Throughout this study, Cls and horizontal inequity indices (HIs) steadily but consistently decreased, suggesting that inequalities and inequities in participation in gastric and colorectal cancer screening were weakened after the implementation of the national public cancer screening program. Decomposition analyses revealed that whereas decreases in inequalities mostly stemmed from income and educational levels; higher income and better education levels are still major contributors to the observed inequalities that influence participation in cancer screening services in Korea.

Conclusion: Our empirical findings suggest that, although the policy of reducing out-of-pocket payment for cancer screening may contribute to the observed decreases in inequality, it alone is not likely to completely eliminate inequality. Further research is required to identify barriers that prevent people with lower socioeconomic status from participation in cancer screening, which allows equal access for equal need.
\end{abstract}

Keywords: Cancer screening, Inequality, Concentration index, Decomposition, Korea, Preventive care

\section{Background}

Cancer screening refers to the use of simple tests across a healthy population to identify individuals who have the disease [1]. It contributes to considerable reduction of both clinical and financial burdens of through early detection and timely treatment for such as colorectal, cervical, and breast cancers [2-4]. For example, the decreased incidence of colorectal cancer during the 1980s and 1990s in the United States is known to be attributed to an increase

\footnotetext{
* Correspondence: jn.hwang@utoronto.ca

${ }^{2}$ Centre for Research on Inner City Health, St. Michael's Hospital, 30 Bond

Street, Toronto, ON M5B 1 W8, Canada

Full list of author information is available at the end of the article
}

in screening services [5]. In Korea, patients screened for gastric cancer appeared to have 30-70 \% lower mortality compared to un-screened patients [6]. Despite the significant clinical benefits of preventive cancer screening services, a concern arises that the rate of screening services are not uniformly utilized over the population $[7,8]$. Cumulative evidence suggests that participation in screening is more concentrated in individuals with higher income for various cancers such as breast, cervical, colorectal and gastric [8-11].

To increase screening and reduce the imbalance in a population, a population screening program at the national level, which establishes guidelines for age-specified 
regular cancer screenings, is recommended [2]. Nationwide screening programs are closely related to overall increases in the use of cancer screening services and decreases in cancer incidence and cancer-related mortality [2]. It is not clear, however, whether these programs facilitate equal use of such services across different income groups [7]. Few studies have examined the impact of the implementation of public cancer screening programs, observing that national-level mass screening may contribute to decreasing inequality in screening participation for breast and cervical cancers [12-16]. Existing evidence is somewhat limited; therefore, the impact of those mass screening policies across socioeconomic status (SES) is not fully understood.

In Korea, the national cancer screening program was implemented in 1999 to provide free screening services for gastric, cervical, and breast cancer to Medicaid enrollees (means-tested program for individuals of lower income); eligible individuals at a certain age receive a letter including detailed information about overall cancer screening procedures. Later, the program expanded the target population to include more low-income people along with Medicaid enrollees; additionally, it included colorectal cancer as a target cancer in 2004 [17]. As of 2006, the program provides free screening services for those in the bottom $50 \%$ of income groups and subsidizes $80 \%$ of the costs for high income groups [17]. Since the implementation of mass screening programs, the rate of cancer screening has steadily increased in Korea [13, 18, 19]; however, differences across income groups receiving the screening are still observed, with $41 \%$ in the lowest income quartile receiving screening compared with $54 \%$ in the highest in 2012 [20].

As there is limited evidence on whether or not the program contributes to encouraging individuals with lower income to utilize the screening, it still remains unanswered if the current mass screening program supports diminishing income-related inequality in cancer screening services. Therefore, this study aimed to examine whether income-related inequalities in cancer screening services exist, and if so, whether the trends of the inequalities has shrunk since the implementation of the national cancer screening program in Korea, 1998-2011. Furthermore, this study assessed the contribution of various socio-demographic factors to the observed inequalities in the use of cancer screening services over the past years.

\section{Methods}

\section{Data source}

Data from Korea National Health and Nutrition Examination Survey (KNHANES) from 1998 (Cycle 1), 2005 (Cycle 3), 2010-2012 (Cycle 5) were utilized. The KNHANES is a nationally representative cross-sectional survey for examining health and nutritional status of Koreans and monitoring health risk factors and the prevalence of chronic disease in the Korean population [21]. Starting from the 2007 survey (Cycle 4), the frequency of the survey has been changed from once every 3 year to every year, and cycle 4 and 5 are composed of a three-year survey [21]. The KNHANES is comprised of non-institutionalized Koreans residing in Korea who are sampled based on a multi-stage clustered probability design, and the survey collects detailed information on socio-demographic information, health behaviours, quality of life, healthcare utilization, and results of various health examination [21]. The KNHANES survey weights are provided in order to represent the entire Korean population by adjusting for complex survey designs, survey non-response and post-stratification [21]. The KNHANES is statistically designed for comparisons between different cycles, and any study using the KNHANES data is generally recommended applying KNHANES survey weights [22]. More information on the KNHANES can be found at http://knhanes.cdc.go.kr/knhanes/eng.

In this study, we included respondents over the age of 40 for gastric cancer $(n=4819$ for cycle 1 (1998), 4580 for cycle 3 (2005), 12274 for cycle 5 (2010-2012)) and over the age of 50 for colorectal cancer $(n=2887$ for cycle 1(1998), 2610 for cycle 3 (2005), 8460 for cycle 5(2010-2012)), based on the screening initiation age for each cancer recommended by the national cancer screening program in Korea [17].

\section{Variables}

\section{Gastric and colorectal cancer screening}

The use of cancer screening services was measured by an individual's self-report as to whether they had received gastric and colorectal cancer screening services. In the KNHANES, each participant was asked "Have you received gastric/colorectal cancer screening services within the past two years?" and the participant recorded their response as "Yes or No".

\section{Socioeconomic status}

To measure the degree of inequality in gastric and colorectal cancer screening participation, equivalised annual household income was used. Income has been considered as a main indicator of SES, and it has been widely used for Concentration Index (CI) and decomposition analysis [23, 24].

\section{Other variables}

For our decomposition models, socio-demographic factors determining participation in cancer screening were selected in order to quantify each contribution of the factors to the observed inequality. These socio-demographic factors were selected based on Andersen's Health Behaviour 
Model and determinants of health care utilization from previous studies [25-27]. The Andersen's Health Behaviour Model is a conceptual model introducing a wide range of factors associated with use of health care services. In this model, the use of health services is determined by three dimensions including predisposing factors (e.g., age, gender, education, employment status, etc.), enabling factors (e.g., income, health insurance, and a regular source of health care, etc.), and need factors (e.g., objective and subjective needs). Based on included various socio-demographic variables for decomposition analysis, we identified need and non-need factors. The need factors (i.e., age (continuous), sex, and self-rated health (good; fair; poor)) generally reflect an individual's health care needs, representing difference in need of health services. The non-need factors include various socioeconomic factors such as marital status (single; married), educational level (elementary school; middle school; high school; university \& above), employment status (manual; non-manual; others including unemployment and out of labour market), income (quintile), region (metro Seoul areas; non-metro Seoul areas), place of residence (urban; rural) and type of national health insurance (NHI, Medicaid; neither).

\section{Statistical analyses}

To measure socioeconomic inequalities in participation in cancer screening services for 1998 (Cycle 1), 2005 (Cycle 3), and 2010-2012 (Cycle 5), we first calculated the CI for each cycle of the KNHANES, a tool which has been widely used to assess income-related inequality in the fields of health economics and policy research [28, 29]. After we obtained the CIs, we employed decomposition approaches to quantify socio-demographic factors contributing to the observed inequalities and its changes in contribution of socio-demographic factors over the past 13 years in relation to both gastric and colorectal screening services in Korea.

\section{Concentration index}

The CI is defined as twice the area between the line of equality (the 45-degree line) and a concentration curve, where the individuals are ranked by socioeconomic levels, generally income and the cumulative rankings of each individual is plotted against the cumulative share of health outcomes or healthcare utilization [30]. The CI represents whether the outcome variable of interest is concentrated more at lower of higher level of income. The outcome variable is concentrated among the rich (the poor), the CI has a positive (negative) value, suggesting "pro-rich" ("propoor"). The CI can be expressed as:

$$
\mathcal{C}=\frac{2}{\mu} \operatorname{cov}\left(y_{i}, r_{i}\right)
$$

Where $y$ is the health care variable, $r$ is the fractional rank in the income distribution and $\mu$ is the mean of the health care variable. The CI is typically bound between -1 and +1 ; however, for binary outcome, the bounds of the $\mathrm{CI}$ depend on the minimum, and the maximum, and the mean of the outcome variable. When the CI is used to compare inequality across time, place and sub-population group, calculating the $\mathrm{CI}$ for binary outcome is potentially problematic because the possible range of the CI value differs by mean of the outcome variable [31-34]. To resolve this issue, the CI needs to be normalized by multiplying (1- mean of the outcome variable), as it referred normalized CI in this study, following the prior studies [31-33].

\section{Decomposition of the $\mathrm{Cl}$ and calculating Horizontal inequity index (HI)}

The basic idea of decomposing the $\mathrm{CI}$ is quantifying each contribution of need and non-need factors to the observed CI because a sum of the contribution from each factor and residuals is the overall CI [35]. Decomposition of the CI can be expressed by the formula:

$$
C=\sum_{k}\left(\frac{\beta_{k} \bar{x}_{k}}{\mu}\right) c_{k}+\frac{C G_{\varepsilon}}{\mu}
$$

Where the index $K$ refers to the regressor included in the underlying equation, $C_{K}$ is the $C I$ for each of the individual regressor, $\beta_{\kappa}$ is the coefficient for each of the determinants, $\bar{x}_{k}$ is the mean of each of the regressor, and $\mu$ is the mean of the health care variable under consideration. $C G_{\varepsilon}$ is the generalized $C$ for the residual from the underlying regression [35]. The residual error term in this equitation represents the inequality in the use of cancer screening services that is not explained by systematic differences [35].

Followed by decomposing the CIs, Horizontal inequity index (HI) was obtained by subtracting all contributions from "need" factors from the overall CI [35]. The $\mathrm{HI}$ is a widely used tool for determining whether or not the observed inequality can be considered as a matter of inequity [35]. A positive (negative) HI indicates a higher share of health care use among the better-off (the worse-off). The HI is based on the idea that access to health services is equitable when individuals have the equal need of the service [35]. Inequality and inequity are used interchangeably in existing literature, but a distinction should be made [36]. While both inequality and inequity indicate difference in health or health care between different population groups, the latter one implies unfairness or injustice that should be modified [36, 37]. 


\section{Decomposition of changes in the $\mathrm{Cl}$}

To understand how each variable contributes to changes in the CI between cycle 1 (KNHANES 1998) and cycle 5 (KNHANES 2010-2012), an Oaxaca-type decomposition of the CI was applied.

$$
\begin{aligned}
C 2-C 1 & =\sum_{k} \eta_{k t 2}\left(C_{k t 2}-C_{k t 1}\right)+\sum_{k} C_{k t 1}\left(\eta_{k t 2}-\eta_{k t 1}\right) \\
& +\left(\frac{G C_{\varepsilon t 2}}{\mu_{t 2}}\right)-\left(\frac{G C_{\varepsilon t 1}}{\mu_{t 1}}\right)
\end{aligned}
$$

Where $C 1$ and $C 2$ are the $C I$ for two different cyclescycle1 (KNHANES 1998) and cycle 5 (KNHANES 20102012), $\eta_{k t}$ is the elasticity for the $k$ regressor at time $t$ (t1:1998 and t2: 2010-2012), $C_{k t}$ is the CI of determinants $k$ at time $t$ and the last two terms constitute the difference in the residuals from decomposition of cycle1 (KNHANES 1998) and cycle 5 (KNHANES 2010-2012) [23].

All analyses were conducted using STATA v. 12, and survey weights provided by KNHANES were applied to all analyses.

\section{Results}

Table 1 shows the total numbers of cancer screening recipients in 1998 (Cycle 1), 2005 (Cycle 3) and 20102012 (Cycle 5). The average rate of colorectal and gastric cancer screening over 14 years was $30.4 \%$ and $40.5 \%$, respectively. The screening rates in both cancer screenings continuously increased, from $7.4 \%$ in 1998 to $42.0 \%$ in 2010-2012 for colorectal cancer and from 13.7 \% in 1998 to $54.0 \%$ in 2010-2012 for gastric cancer. The descriptive characteristics of the respondents by cancer screening services are found in Additional file 1 (Tables S1 and S2).

The concentration indices (CIs) for cancer screening services are shown in Fig. 1 and Fig. 2. Positive values of the CI observed consistently over time indicates that more use of cancer screening services has been concentrated among individuals with higher income for both cancers, but the degree of the CIs has slightly decreased

Table 1 A total sample of cancer screening recipients by KNHANES cycle 1 (1998), cycle 3 (2005), cycle 5 (2010-2012)*

\begin{tabular}{llllll}
\hline Colorectal cancer & Yes & $\%$ & No & $\%$ & Total \\
\hline Cycle 1 (1998) & 214 & 7.4 & 2663 & 92.6 & 2877 \\
Cycle 3 (2005) & 475 & 18.2 & 2135 & 81.8 & 2610 \\
Cycle 5 (2010-2012) & 3556 & 42.0 & 4904 & 58.0 & 8460 \\
Gastric cancer & Yes & $\%$ & No & $\%$ & Total \\
Cycle 1 (1998) & 660 & 13.7 & 4159 & 86.3 & 4819 \\
Cycle 3 (2005) & 1490 & 32.5 & 3090 & 67.5 & 4580 \\
Cycle 5 (2010-2012) & 6624 & 54.0 & 5650 & 46.0 & 12274 \\
\hline
\end{tabular}

"The KNAHNES response rate: cycle 1 (1998): $85.6 \%$; cycle 3 (2005): $92.8 \%$; cycle 5 (2010-2012): $80.8 \%$

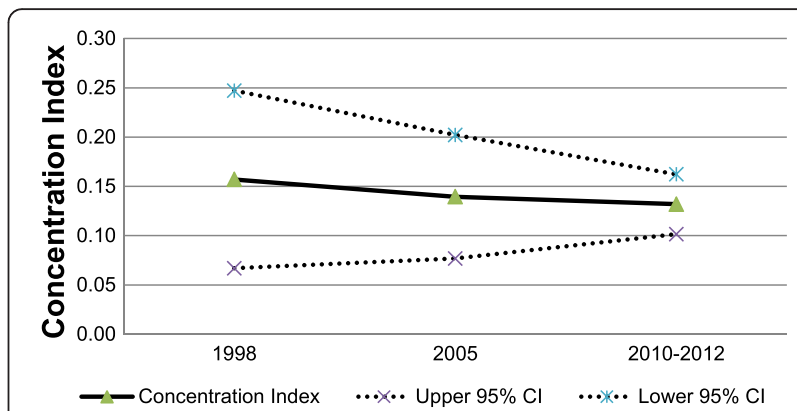

Fig. 1 Concentration Index ${ }^{*}$ for colorectal cancer screening services with $95 \%$ confidence interval, Korea National Health and Nutrition Examination Survey (KNHANES) cycle 1 (1998), cycle 3 (2005), and cycle 5 (2010-2012). ${ }^{*}$ Concentration index presented in this figure are normalized concentration indices (Wagstff, 2005;2011)

for gastric cancer from 0.179 (95 \% CI: $0.128-0.230$ ) in 1998 to 0.132 (95 \% CI: $0.107-0.158$ ) in 2010-2012. The CIs for the use of colorectal cancer screening also have decreased to 0.131 (95 \% CI: 0.102-0.162) in 2010-2012 from 0.157 (95 \% CI: 0.067-0.247) in 1998.

Table 2 and Table 3 present the decomposition of the CIs in colorectal cancer screening services and gastric cancer screening services for 1998 and 2010-2012, respectively. Elasticity for each factor $\left(\frac{\beta_{k} \bar{x}_{k}}{\mu}\right)$ in the first column shows the sensitivity of cancer screening services with respect to each factor. The CI for each factor is presented in the second column. This indicates the distribution of each factor by income levels. For instance, positive $\mathrm{C}_{k}$ value (second column of each year) for highest education (Education - completion of University or above) in Tables 2 and 3 indicates that highest educational attainment is more concentrated in the rich. Lastly, contribution $\left(\left(\frac{\beta_{k} \bar{x}_{k}}{\mu}\right) C_{k}\right)$ in the third column shows how much each factor contributes to inequality (the overall $\mathrm{CI}$ ) in the use of cancer screening services. Positive contribution of a

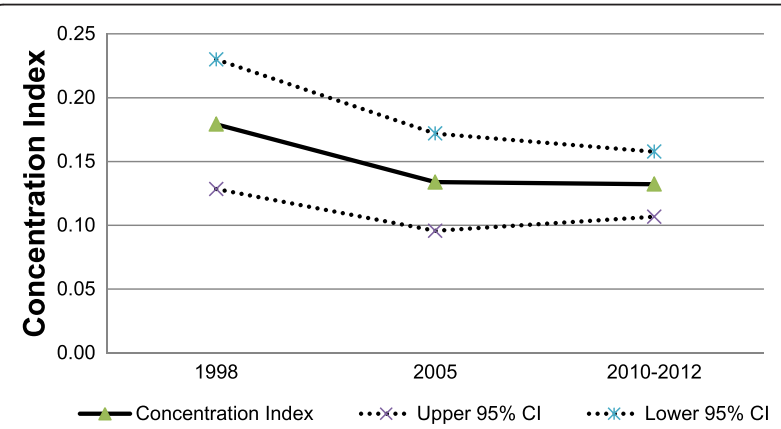

Fig. $\mathbf{2}$ Concentration Index ${ }^{*}$ for gastric cancer screening services with $95 \%$ confidence interval, Korea National Health and Nutrition Examination Survey (KNHANES) cycle 1 (1998), cycle 3 (2005), and cycle 5 (2010-2012). * Concentration index presented in this figure are normalized concentration indices (Wagstff, 2005;2011) 
Table 2 Results from decomposition analysis for participation of colorectal cancer screening services among Korean adults, Korea National Health and Nutrition Examination Survey (KNHANES) cycle 1 (1998), cycle 3 (2005), and cycle 5 (2010-2012)

\begin{tabular}{|c|c|c|c|c|c|c|c|c|c|}
\hline \multirow[t]{2}{*}{ Colorectal cancer screening } & \multicolumn{3}{|c|}{ Cycle 1 (1998) } & \multicolumn{3}{|c|}{ Cycle 3 (2005) } & \multicolumn{3}{|c|}{ Cycle 5 (2010-2012) } \\
\hline & Elasticity & $C_{k}$ & Contribution & Elasticity & $C_{k}$ & Contribution & Elasticity & $C_{k}$ & Contribution \\
\hline Sex-female & 0.039 & -0.016 & -0.001 & -0.198 & -0.024 & 0.005 & -0.009 & -0.024 & 0.000 \\
\hline Age & -0.636 & -0.017 & 0.011 & -0.083 & -0.029 & 0.002 & -0.264 & -0.039 & 0.010 \\
\hline Self-rated health - fair & -0.076 & 0.102 & -0.008 & -0.088 & 0.088 & -0.008 & 0.037 & 0.040 & 0.001 \\
\hline Self-rated - poor & -0.097 & 0.033 & -0.003 & -0.094 & 0.176 & -0.017 & 0.009 & 0.127 & 0.001 \\
\hline Sub-total - need factors & & & -0.001 & & & -0.017 & & & 0.013 \\
\hline Marital status- married & 0.030 & 0.041 & 0.001 & -0.056 & 0.082 & -0.005 & 0.113 & 0.090 & 0.010 \\
\hline Education - completion of middle school & 0.024 & 0.142 & 0.003 & 0.060 & 0.120 & 0.007 & 0.026 & 0.090 & 0.002 \\
\hline Education - completion of high school & 0.138 & 0.263 & 0.036 & 0.012 & 0.232 & 0.003 & 0.033 & 0.222 & 0.007 \\
\hline Education - completion of university or above & 0.045 & 0.501 & 0.023 & 0.010 & 0.397 & 0.004 & 0.019 & 0.400 & 0.008 \\
\hline Employment - non-manual job & 0.014 & 0.760 & 0.011 & -0.003 & 0.657 & -0.002 & 0.003 & 0.493 & 0.001 \\
\hline Employment-others $^{\mathrm{a}}$ & 0.039 & -0.177 & -0.007 & -0.054 & -0.240 & 0.013 & 0.010 & -0.160 & -0.002 \\
\hline Income quintile 2 (low) & 0.118 & -0.349 & -0.041 & 0.062 & -0.319 & -0.020 & 0.027 & -0.447 & -0.012 \\
\hline Income quintile 3 & 0.032 & 0.031 & 0.001 & 0.045 & 0.060 & 0.003 & 0.039 & -0.057 & -0.002 \\
\hline Income quintile 4 & 0.037 & 0.508 & 0.019 & 0.103 & 0.401 & 0.041 & 0.028 & 0.366 & 0.010 \\
\hline Income quintile 5(high) & 0.099 & 0.910 & 0.090 & 0.112 & 0.830 & 0.093 & 0.039 & 0.799 & 0.031 \\
\hline Health insurance - National Health Insurance & -0.294 & 0.030 & -0.009 & 0.239 & 0.042 & 0.010 & 0.106 & 0.025 & 0.003 \\
\hline Region - Metro Seoul areas & 0.061 & 0.159 & 0.010 & 0.020 & 0.156 & 0.003 & 0.013 & 0.076 & 0.001 \\
\hline Place of residence - rural areas & -0.247 & -0.052 & 0.013 & 0.143 & -0.044 & -0.006 & -0.151 & -0.033 & 0.005 \\
\hline Sub-total - non-need factors & & & 0.150 & & & 0.144 & & & 0.063 \\
\hline Residual & & & 0.008 & & & 0.012 & & & 0.055 \\
\hline $\begin{array}{l}\text { Normalized concentration index (Cl) } \\
\text { (95\% confidence interval) }\end{array}$ & & $0.157(C$ & $.067-0.247)$ & & $0.139(C$ & $.077-0.202)$ & & 0.131( & $.102-0.162)$ \\
\hline
\end{tabular}

a "Others" included unemployment and out of labour market

certain factor shows the factor contributes to the measured "pro-rich" inequalities in cancer screening services. For colorectal cancer screening, highest income quintile (income quintile 5) was consistently the largest contributor to the overall CIs over the past years (Table 2). Higher education attainment (completion of high school, and university or above) was the second largest contributor to the pro-rich patent of the CI in 1998 and in 2010-2102 (Table 2).

Higher income (income quintile 4 and 5) was the major contributor to the existing inequality in the use of gastric cancer screening services (Table 3). Completion of university or above and non-manual job also positively contributed to the pro-rich overall CIs in 1998 and 2010-2012 (Table 3).

For horizontal inequity index (HI), obtained by subtracting a sub total contribution of need factors from the overall CI, positive values of HIs for colorectal cancer screening services were consistently observed in the past years (1998: 0.158; 2005:0.157; 2010-2012: 0.118). Although the degree of the $\mathrm{HI}$ has steadily decreased, inequity in the use of colorectal cancer screening still persisted (Fig. 3). For the use of gastric cancer screening, a similar pattern was observed, suggesting a persistence of inequity between 1998 (HI: 0.182) and 2010-2012 (HI: 0.146), but the magnitude has become relatively smaller compared to the year of 1998 (Fig. 3).

To understand what factors have contributed to changes in the CIs in the use of both cancer screening services between cycle 1 (1998) and cycle 5 (20102012), we conducted Oaxaca type-decomposition for changes in the $\mathrm{CI}$ as an additional analysis. Tables 4 and 5 show the changes in the CIs in colorectal and gastric cancer screening services. The first column shows changes in magnitude of inequality in the contributors and the second column shows changes in the elasticity of the cancer screening services with respect to these contributors. The total changes for each determinant and the percent changes are presented in the last two columns. Overall, changing elasticity than changing CIs were more likely to contribute to the reduction in the 
Table 3 Results from decomposition analysis for participation of gastric cancer screening services among Korean adults, Korea National Health and Nutrition Examination Survey (KNHANES) cycle 1 (1998), cycle 3 (2005), and cycle 5 (2010-2012)

\begin{tabular}{|c|c|c|c|c|c|c|c|c|c|}
\hline \multirow[t]{2}{*}{ Gastric cancer screening } & \multicolumn{3}{|l|}{1998} & \multicolumn{3}{|l|}{2005} & \multicolumn{3}{|c|}{ 2010-2012 } \\
\hline & Elasticity & $C_{k}$ & Contribution & Elasticity & $C_{k}$ & Contribution & Elasticity & $C_{k}$ & Contribution \\
\hline Sex-female & -0.072 & -0.015 & 0.001 & 0.031 & -0.020 & -0.001 & 0.097 & -0.018 & -0.002 \\
\hline Age & -0.250 & -0.037 & 0.009 & -0.169 & -0.050 & 0.008 & 0.295 & -0.052 & -0.015 \\
\hline Self-rated health - fair & -0.079 & 0.090 & -0.007 & -0.045 & 0.069 & -0.003 & 0.026 & 0.026 & 0.001 \\
\hline Self-rated - poor & -0.132 & 0.041 & -0.005 & -0.069 & 0.172 & -0.012 & 0.015 & 0.119 & 0.002 \\
\hline Sub-total - need factors & & & -0.002 & & & -0.007 & & & -0.014 \\
\hline Marital status- married & 0.086 & 0.060 & 0.005 & 0.101 & 0.097 & 0.010 & 0.145 & 0.084 & 0.012 \\
\hline Education - completion of middle school & 0.026 & 0.034 & 0.001 & 0.013 & -0.013 & 0.000 & 0.017 & -0.049 & -0.001 \\
\hline Education - completion of high school & 0.084 & 0.185 & 0.016 & -0.032 & 0.144 & -0.005 & 0.025 & 0.133 & 0.003 \\
\hline Education - completion of university or above & 0.077 & 0.463 & 0.036 & -0.002 & 0.431 & -0.001 & 0.018 & 0.347 & 0.006 \\
\hline Employment - non-manual job & 0.051 & 0.564 & 0.029 & 0.031 & 0.568 & 0.017 & 0.013 & 0.382 & 0.005 \\
\hline Employment - others $^{a}$ & 0.073 & -0.120 & -0.009 & 0.003 & -0.313 & -0.001 & -0.012 & -0.185 & 0.002 \\
\hline Income quintile 2 (low) & 0.079 & -0.424 & -0.033 & 0.063 & -0.366 & -0.023 & 0.019 & -0.468 & -0.009 \\
\hline Income quintile 3 & 0.053 & -0.063 & -0.003 & 0.033 & 0.040 & 0.001 & 0.022 & -0.053 & -0.001 \\
\hline Income quintile 4 & 0.142 & 0.420 & 0.060 & 0.037 & 0.404 & 0.015 & 0.039 & 0.384 & 0.015 \\
\hline Income quintile 5(high) & 0.066 & 0.869 & 0.058 & 0.106 & 0.821 & 0.087 & 0.044 & 0.810 & 0.036 \\
\hline Health insurance - National Health Insurance & -0.034 & 0.025 & -0.001 & -0.181 & 0.036 & -0.007 & 0.031 & 0.024 & 0.001 \\
\hline Health insurance - neither & -0.008 & -0.327 & 0.003 & -0.002 & -0.565 & 0.001 & 0.000 & -0.184 & 0.000 \\
\hline Region - Metro Seoul areas & 0.031 & 0.122 & 0.004 & 0.016 & 0.113 & 0.002 & -0.009 & 0.044 & 0.000 \\
\hline Place of residence - rural areas & -0.116 & -0.063 & 0.007 & 0.095 & -0.038 & -0.004 & -0.005 & -0.033 & 0.000 \\
\hline Sub-total - non-need factors & & & 0.170 & & & 0.094 & & & 0.060 \\
\hline Residual & & & 0.011 & & & 0.047 & & & 0.077 \\
\hline $\begin{array}{l}\text { Normalized concentration index (Cl) } \\
\text { (95\% confidence interval) }\end{array}$ & & 0.179 & $.128-0.230)$ & & 0.134( & $0.096-0.172)$ & & 0.132 & $.107-0.158)$ \\
\hline
\end{tabular}

a"Others" included unemployment and out of labour market

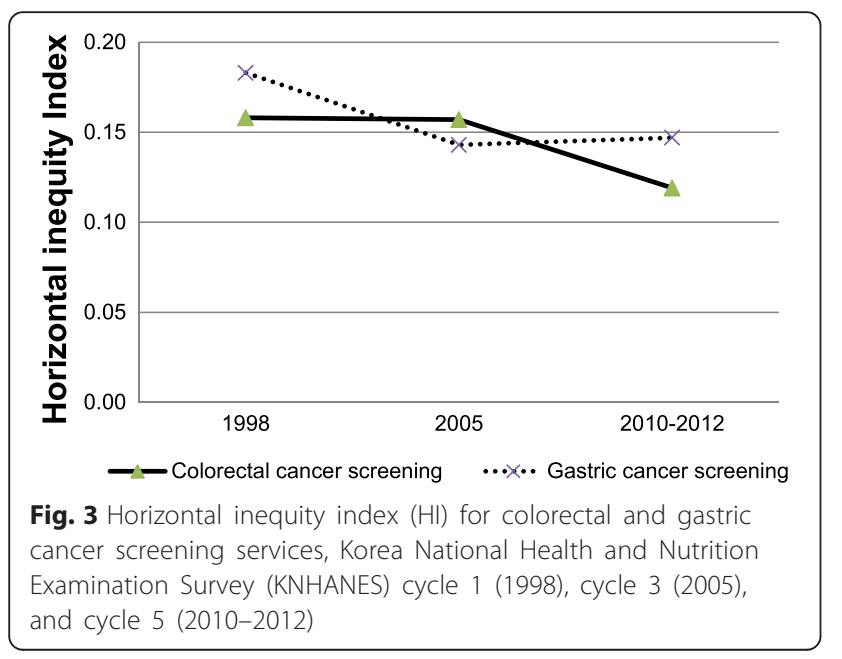

pro-rich inequalities in cancer screening services. For colorectal cancer screening services, differences in higher education, and higher income were largely responsible for a decrease in inequality. With respect to gastric cancer screening services, the largest determinants for changes in inequality were also from income and education levels. It is interesting to note that being out of labor market and unemployed contributed to increasing inequalities in gastric and colorectal cancer screening services.

\section{Discussion}

This study attempted to measure income-related inequalities in the use of gastric and colorectal cancer screening services by estimating the concentration index (CI), and also quantified each contribution from various socio-demographic factors such as educational and employment status to the observed inequalities by decomposing the CI over the past decades. The CIs for the use of cancer screening services appeared to decrease from 0.157 to 0.131 in colorectal cancer and from 0.179 to 0.132 in gastric cancer, which reveals a steady but 
Table 4 Oaxaca-type decomposition for changes in the Cls in colorectal cancer screening services between Korea National Health and Nutrition Examination Survey (KNHANES) cycle 1(1998) and cycle 5 (2010-2012)

\begin{tabular}{|c|c|c|c|c|}
\hline \multirow{3}{*}{$\begin{array}{l}\text { Oaxaca-type approach for colorectal } \\
\text { cancer screening }\end{array}$} & \multicolumn{4}{|c|}{ Between cycle 1(1998) and cycle 5 (2010-2012) } \\
\hline & \multicolumn{4}{|c|}{ Changes arising from change in } \\
\hline & $C_{k}$ & Elasticity & Total & Percentage \\
\hline Sex-female & 0.0001 & 0.0008 & 0.0009 & -3.33 \\
\hline Age & 0.0058 & -0.0062 & -0.0004 & 1.50 \\
\hline Self-rated health - fair & -0.0024 & 0.0116 & 0.0092 & -35.88 \\
\hline Self-rated - poor & 0.0009 & 0.0035 & 0.0044 & -17.14 \\
\hline Marital status- married & 0.0056 & 0.0034 & 0.0090 & -35.20 \\
\hline Education - completion of middle school & -0.0014 & 0.0003 & -0.0010 & 3.98 \\
\hline Education - completion of high school & -0.0014 & -0.0275 & -0.0288 & 112.44 \\
\hline Education - completion of University or above & -0.0019 & -0.0134 & -0.0153 & 59.48 \\
\hline Employment - non-manual job & -0.0007 & -0.0085 & -0.0093 & 36.19 \\
\hline Employment - others $^{\mathrm{a}}$ & 0.0002 & 0.0051 & 0.0052 & -20.40 \\
\hline Income quintile 2 (low) & -0.0026 & 0.0317 & 0.0291 & -113.51 \\
\hline Income quintile 3 & -0.0034 & 0.0002 & -0.0032 & 12.51 \\
\hline Income quintile 4 & -0.0040 & -0.0045 & -0.0085 & 33.04 \\
\hline Income quintile 5(high) & -0.0043 & -0.0545 & -0.0588 & 229.18 \\
\hline Health insurance - National Health Insurance & -0.0005 & 0.0119 & 0.0114 & -44.30 \\
\hline Region - Metro Seoul areas & -0.0010 & -0.0077 & -0.0088 & 34.27 \\
\hline Place of residence - rural areas & -0.0028 & -0.0048 & -0.0079 & 30.80 \\
\hline Sub-total & -0.0139 & -0.0608 & -0.0727 & 283.64 \\
\hline Residual & & & 0.0471 & -183.64 \\
\hline Total & -0.0251 & & -0.0256 & 100.00 \\
\hline
\end{tabular}

a"Others" included unemployment and out of labour market

consistent decline in income-related inequalities in participation in gastric and colorectal cancer screening services over times in Korea. In addition, Horizontal inequity Index (HI), indicating whether the services participation was equitable across the population after need-adjustment, also consistently persisted, suggesting that the observed inequalities between 1998 and 2010-2012 may be a matter of inequities, avoidable differences in participation in colorectal and gastric cancer screening services.

A plausible explanation for the observed decline in inequalities and inequities of both gastric and colorectal screening services is expanding coverage of free of charge screening services for larger population groups. Out-ofpocket (OOP) payments at the service offered in Korea's National Health Insurance (NHI) have been widely reported as a major barrier to preventive health care services [38], and minimizing individual's financial burdens by expanding the national cancer screening program may result in increasing accessibility of both cancer screening services, in particular among lower income groups.
Results from Oaxaca decomposition of the CIs reassure us that the contributor that is most responsible for decreasing inequalities is income. Income levels have been identified as a main factor driving unequal patterns of preventive cancer screening participation [26, 39]. Previous studies indicated that preventive screening programs, including nationwide population-based screening, is likely to facilitate utilization of the services from individuals with lower SES because the program minimizes possible barriers such as cost, time off work, and information $[7,40,41]$. The consistency of this finding implies that establishing or expanding public cancer screenings under universal health coverage may need to be considered when increasing participation rate of and reducing inequalities for individuals of lower SES.

Even though the CI and HI provide evidence on the degree of existing inequality and inequity with respect to the use of cancer screening services, a question related to its interpretation of the magnitude can been raised. To provide a better understanding of the values, we compared results with previous studies using the same methods. For instance, a recent UK study on colorectal 
cancer screening reported a pro-rich pattern with 0.16 of the CI value [27]. This value is similar to our 1998's CI value but slightly larger than our 2010-2012's CIs for both colorectal and gastric cancer screening services. Compared to other European countries such as Germany (0.126), France (0.135), and Belgium (0.04) [42], the CIs for both colorectal and gastric cancer screenings in Korea have become similar, but still show the persistence of inequalities and inequities. This may imply that the national screening program contributes to decreasing inequalities and inequities in participation in cancer screenings, whereas other national or institutional features may also account for these differences in the degree of inequality (inequity) in cancer screening. In fact, a previous study that measured the CIs in both public and private cancer screenings in Korea suggested different patterns of using cancer screening in different income groups; individuals with higher income were concentrated in the use of private cancer screening at their own cost (CI: 0.143) whereas individuals with lower income were more concentrated in use of public cancer screening services (CI:-0.118) [26, 39].

Although results from our additional analysis (Oaxacatype decomposition for changes in the CIs) indicates that decreases in the inequalities stemmed from income and educational levels, results from the decomposition analyses in 2010-2012 reveal that higher income and better education levels still remain as major contributors to inequalities in participation in cancer screening services in Korea. Consistent with our study, a previous study investigating the utilization of cervical screening across different countries reported that income and education substantially contributes to causing inequalities despite the existence of national policy ensuring equal access or delivery of preventive services [2]. Also, it has been reported that less-educated women were less likely to utilize mammography even after the implementation of a Belgian national screening program that offers free mammography services, although there was an overall decrease of inequalities in cancer screening [14]. These findings from previous studies, along with our results, imply that the implementation of cancer screening programs at the national level could reduce the financial burden of individuals with lower income and educational levels, but it may fail to achieve equal utilization for all population groups.

To fully understand why inequalities still remain after the expansion of public cancer screening services in Korea, further investigation is required. One plausible explanation is that while an individual's economic condition, mainly income, represents ability to pay for services, utilization of cancer screening services may be influenced by numerous factors correlated to income [3, 40, 43-46]. For example, people with lower income and precarious employment may have more difficulties in leaving work to seek preventive care services [47, 48]. Further, considering a profound relationship between income and educational level, lack of knowledge of health services -leading to low levels of health literacy- have an influence on informed decision making about screening $[46,49-51]$ as an individual may underestimate the risk of cancer and the benefits of preventive screening $[7,46,49,50,52]$.

Because income appears to be such an important contributor, policy makers could further focus on understanding the income-cancer screening relationship if they aim to continually reduce the observed inequalities under the current public mass cancer screening program. According to our findings, a greater investment in public cancer screening at the national level is likely to increase not only the total rate of screening, but also can be potentially beneficial in diminishing income-related inequality in the uptake of cancer screening services. The recent national statistics indicates income inequality measured by Gini coefficient has steadily increased over the past decade [53]. This implies that income-related inequalities in participation of cancer screening services could become worse again if no effort to maintain or expand the current public cancer screening is made.

Nevertheless, it is worthwhile to caution that eliminating only financial barriers, without considering further policies to reduce inequality, could be more beneficial to higher SES [48]. In fact, our results from the Oaxaca-type decomposition analyses indicate that pro-rich patterns in those currently unemployed or out of labour market increased along with residual error indicating unexplained component by the included need and non-need factors for both screening services (Tables 4 and 5). Considering this result, policy actions for increasing cancer screening services among those currently unemployed and out of labour market need to be considered. Furthermore, other factors prohibiting individuals of lower income from undertaking screening services need to be further investigated.

Our study has several limitations. First, we did not consider specific intervals for screening modality for colorectal cancer. Although the guideline recommends people to take FOBT every year, to have a sigmoidoscopy within five years, or a colonoscopy within 10 years [17], only KNHANES cycle 4 (2007) and cycle 5 (2010-2012) include information about types of colorectal cancer screening [21]. Second, we compared the lowest-income quintile with the highestincome quintile under the premise that the poor would experience an increase in the uptake of screening as much as the rich do. Although there might be a problem of over-screening among the rich, our premise would be still valid because this study used a survey question asking individual's participation in screening services over the past two years as the outcome measure, not the number of participation [54]. 
Table 5 Oaxaca-type decomposition for changes in the Cls in gastric cancer screening services between Korea National Health and Nutrition Examination Survey (KNHANES) cycle 1(1998) and cycle 5 (2010-2012)

\begin{tabular}{|c|c|c|c|c|}
\hline \multirow{3}{*}{$\begin{array}{l}\text { Oaxaca-type approach for gastric cancer } \\
\text { screening }\end{array}$} & \multicolumn{4}{|c|}{ Between cycle 1(1998) and cycle 5 (2010-2012) } \\
\hline & \multicolumn{4}{|c|}{ Change arising from change in } \\
\hline & $C_{k}$ & Elasticity & Total & Percentage \\
\hline Sex-female & -0.0003 & -0.0025 & -0.0028 & 5.81 \\
\hline Age & -0.0043 & -0.0203 & -0.0245 & 51.38 \\
\hline Self-rated health - fair & -0.0017 & 0.0095 & 0.0078 & -16.35 \\
\hline Self-rated - poor & 0.0012 & 0.0060 & 0.0072 & -15.04 \\
\hline Marital status- married & 0.0035 & 0.0035 & 0.0070 & -14.57 \\
\hline Education - completion of middle school & -0.0014 & -0.0003 & -0.0017 & 3.53 \\
\hline Education - completion of high school & -0.0013 & -0.0110 & -0.0123 & 25.78 \\
\hline Education - completion of University or above & -0.0021 & -0.0276 & -0.0297 & 62.10 \\
\hline Employment - non-manual job & -0.0023 & -0.0218 & -0.0241 & 50.51 \\
\hline Employment - others $^{\mathrm{a}}$ & 0.0008 & 0.0102 & 0.0109 & -22.90 \\
\hline Income quintile 2 (low) & -0.0008 & 0.0255 & 0.0247 & -51.65 \\
\hline Income quintile 3 & 0.0002 & 0.0019 & 0,0021 & -4.48 \\
\hline Income quintile 4 & -0.0014 & -0.0433 & -0.0448 & 93.73 \\
\hline Income quintile 5(high) & -0.0026 & -0.0190 & -0.0216 & 45.23 \\
\hline Insurance - National Health Insurance & 0.0000 & 0.0016 & 0.0016 & -3.34 \\
\hline Insurance - neither & 0.0000 & -0.0026 & -0.0026 & 5.54 \\
\hline Region - Metro Seoul areas & 0.0007 & -0.0048 & -0.0041 & 8.64 \\
\hline Place of residence - rural areas & -0.0001 & -0.0066 & -0.0068 & 14.45 \\
\hline Sub-total & -0.0120 & -0.1017 & -0.1109 & 232.25 \\
\hline Residual & & & 0.0632 & -132.25 \\
\hline Total & -0.0470 & & -0.0478 & 100.00 \\
\hline
\end{tabular}

a"Others" included unemployment and out of labour market

\section{Conclusions}

This study provides evidence on changes in socioeconomic inequalities in participation in gastric and colorectal cancer screening in Korea over the past decade, and shows decreases in inequalities for both cancer screenings. Despite policy reducing out-of-pocket payment for cancer screening possibly contributing to observed decreases in inequality, it alone is not likely to eliminate inequalities. Further research needs to identify barriers that prevent low-income people from the uptake of cancer screening despite free cancer screening program for all individuals who are eligible for Korea's National Health Insurance.

\section{Additional file}

Additional file 1: Table S1. Basic characteristics of respondents by participation of colorectal cancer screening ${ }^{\S}$, KNHANES cycle 1 (1998), cycle 3 (2005) and cycle 5 (2010-2012). Table S2. Basic characteristics of the respondents by participation of gastric cancer screening ${ }^{\S}$, KNHANES cycle 1 (1998), cycle 3 (2005) and cycle 5 (2010-2012). (DOCX 31 kb)

\section{Competing interests}

The authors declare no conflict of interest.
Authors' contributions

SK and $\mathrm{JH}$ conceptualized the study, interpreted the results and drafted the manuscript. JH conducted data analysis and provided critical reviews. SK and $\mathrm{JH}$ revised and prepared final version of the manuscript. Both authors read and approved the final manuscript.

\section{Author details}

${ }^{1}$ Takemi program in International Health, Harvard School of Public Health, Boston, MA, USA. ${ }^{2}$ Centre for Research on Inner City Health, St. Michael's Hospital, 30 Bond Street, Toronto, ON M5B 1 W8, Canada.

Received: 12 October 2015 Accepted: 11 February 2016

Published online: 24 February 2016

\section{References}

1. WHO: National cancer control programmes- Policies and managerial guidelines. WHO ed., 2nd edition: World Health Organization 2002.

2. McKinnon B, Harper S, Moore S. Decomposing income-related inequality in cervical screening in 67 countries. Int J Public Health. 2011;56:139-52.

3. Berrino F, De Angelis R, Sant M, Rosso S, Bielska-Lasota M, Coebergh JW, et al. Survival for eight major cancers and all cancers combined for European adults diagnosed in 1995-99: results of the EUROCARE-4 study. Lancet Oncol. 2007;8:773-83.

4. Provenzale D, Gray RN. Colorectal cancer screening and treatment: review of outcomes research. J Natl Cancer Inst Monogr 2004:45-55.

5. Kamangar F, Dores GM, Anderson WF. Patterns of cancer incidence, mortality, and prevalence across five continents: defining priorities to reduce cancer disparities in different geographic regions of the world. J Clin Oncol. 2006;24: 2137-50. 
6. Lee H, Min BH, Lee JH, Son HJ, Kim JJ, Rhee JC, et al. Survival outcome associated with the screening interval for gastric cancer in Korea. Digestion. 2011;84:142-8

7. Palencia L, Espelt A, Rodriguez-Sanz M, Puigpinos R, Pons-Vigues M, Pasarin $\mathrm{Ml}$, et al. Socio-economic inequalities in breast and cervical cancer screening practices in Europe: influence of the type of screening program. Int J Epidemiol. 2010;39:757-65.

8. Lee K, Lim HT, Hwang SS, Chae DW, Park SM. Socio-economic disparities in behavioural risk factors for cancer and use of cancer screening services in Korean adults aged 30 years and older: the Third Korean National Health and Nutrition Examination Survey, 2005 (KNHANES III). Public Health. 2010; 124:698-704.

9. Whynes DK, Frew EJ, Manghan CM, Scholefield JH, Hardcastle JD. Colorectal cancer, screening and survival: the influence of socio-economic deprivation. Public Health. 2003;117:389-95.

10. Hao Y, Jemal A, Zhang X, Ward EM. Trends in colorectal cancer incidence rates by age, race/ethnicity, and indices of access to medical care, 1995-2004 (United States). Cancer Causes Control. 2009;20:1855-63.

11. Katz SJ, Hofer TP. Socioeconomic disparities in preventive care persist despite universal coverage: breast and cervical cancer screening in Ontario and the United States. JAMA. 1994;272:530-4.

12. De Maio FG, Linetzky B, Ferrante $D$. Changes in the social gradients for Pap smears and mammograms in Argentina: evidence from the 2005 and 2009 National Risk Factor Surveys. Public Health. 2012;126:821-6.

13. Lee M, Park EC, Chang HS, Kwon JA, Yoo KB, Kim TH. Socioeconomic disparity in cervical cancer screening among Korean women: 1998-2010. BMC Public Health. 2013;13:553.

14. Puddu M, Demarest S, Tafforeau J. Does a national screening programme reduce socioeconomic inequalities in mammography use? Int J Public Health. 2009;54:61-8.

15. Puigpinos-Riera R, Serral G, Pons-Vigues M, Palencia L, Rodriguez-Sanz M, Borrell C. Evolution of inequalities in breast and cervical cancer screening in Barcelona: population surveys 1992, 2001, and 2006. J Womens Health. 2011;20:1721-7

16. Tabuchi T, Hoshino T, Nakayama T, Ito Y, loka A, Miyashiro I, et al. Does removal of out-of-pocket costs for cervical and breast cancer screening work? A quasi-experimental study to evaluate the impact on attendance, attendance inequality and average cost per uptake of a Japanese government intervention. Int J Cancer. 2013;133:972-83.

17. Korea Ministry of Health and Welfare. National cancer screening programmes guidelines. Seoul: Ministry of Health and Welfare; 2006.

18. Suh M, Choi KS, Lee YY, Jun JK. Trends in cancer screening rates among korean men and women: results from the Korean National Cancer Screening Survey, 2004-2012. Cancer Res Treat. 2013;45:86-94.

19. Choi KS, Jun JK, Lee HY, Hahm Ml, Oh JH, Park EC. Increasing uptake of colorectal cancer screening in Korea: a population-based study. BMC Public Health. 2010;10:265.

20. Korea Ministry of Health and Welfare. Trend in the uptake of cancer screening: adults over the age of 19. Seoul: Korea Ministry of Health and Welfare; 2013.

21. Kweon S, Kim Y, Jang M-j, Kim Y, Kim K, Choi S, et al. Data resource profile: the Korea National Health and Nutrition Examination Survey (KNHANES). Int J of Epidemiology. 2014;43:69-77.

22. Korea Centres for Disease and Control and Prevention (KCDC): The fifth Korea National Health and Nutrition Examination Survey Data User Guide. KCDC 2012

23. Wagstaff A, van Doorslaer E, Watanabe N. On decomposing the causes of health sector inequalities with an application to malnutrition inequalities in Vietnam. J Econom. 2003;112:207-23.

24. van Doorslaer E, Masseria C, Koolman X, the OECD Health Equity Research Group. Inequalities in access to medical care by income in developed countries. CMAJ. 2006;174:177-83.

25. Andersen RM. Revisiting the behavioral model and access to medical care: does it matter? J Health Soc Behav. 1995;36(1):1-10.

26. Heo J, Hwang J. Income-related Inequalitiesin cancer screening in Korea: using Concentration Index $(\mathrm{Cl})$ and decomposition of $\mathrm{Cl}$. Health Soc Welfare Rev. 2014;34:59-81.

27. Solmi F, Von Wagner C, Kobayashi LC, Raine R, Wardle J, Morris S. Decomposing socio-economic inequality in colorectal cancer screening uptake in England. Soc Sci Med. 2015;134:76-86.
28. O'Donnell O, van Dooslaer E, Wagstaff A, Lindelow M. Analyzing Health Equity Using Household Survey Data: A Guide to Techniques and Their Impementation. Washington, DC: The World Bank; 2008.

29. Haper SL, Lynch J. Methods for measuring cancer disparities : using data relevant to healthy people 2010 cancer-related objectives. Bethesda, MD: National Cancer Institute; 2005.

30. O'Donnell O, van Dooslaer E, Wagstaff A, Lindelow M. Chapter 8. The Concentration Index. In: Analyzing Health Equity Using Household Survey Data : A Guide to Techniques and Their Impementation. Washington, DC: The World Bank; 2008. p. 95-108.

31. Wagstaff $A$. The concentration index of a binary outcome revisited. Health Econ. 2011;20:1155-60.

32. Wagstaff A. The bounds of the concentration index when the variable of interest is binary, with an application to immunization inequality. Health Econ. 2005:14:429-32.

33. Devaux M. Income-related inequalities and inequities in health care services utilisation in 18 selected OECD countries. Eur J Health Econ. 2015;16:21-33.

34. Hajizadeh M, Karen Campbell M, Sarma S. Socioeconomic inequalities in adult obesity risk in Canada: trends and decomposition analyses. Eur J Health Econ. 2014;15:203-21.

35. O'Donnell O, van Doorslear E, Wagstaff A, Lindelow M. Chapter 15. Measuring and explaining inequity in health service delivery. In: Analyzing Health Equity Using Household Survey Data : A Guide to Techniques and Their Impementation. Washington, DC: The World Bank; 2008. p. 177-86.

36. Kawachi I, Subramanian SV, Almeida-Filho N. A glossary for health inequalities. J Epidemiol Community Health. 2002;56:647-52.

37. Hwang J, Rudnisky C, Bowen S, Johnson JA. Income-related inequalities in visual impairment and eye screening services in patients with type 2 diabetes. J Public Health. 2015. doi:10.1093/pubmed/fdv185.

38. Kwon S. Thirty years of national health insurance in South Korea: lessons for achieving universal health care coverage. Health Policy Plan. 2009;24:63-71.

39. Devaux M, De Looper M. Income-related inequalities in health service utilisation in 19 OECD countries, 2008-2009. OECD Health Working Papers No. 58, OECD Publishng 2012.

40. von Wagner C, Baio G, Raine R, Snowball J, Morris S, Atkin W, et al. Inequalities in participation in an organized national colorectal cancer screening programme: results from the first 2.6 million invitations in England. Int J Epidemiol. 2011;40:712-8.

41. Baker D, Middleton E. Cervical screening and health inequality in England in the 1990s. J Epidemiol Community Health. 2003;57:417-23.

42. Carrieri $V$, Wuebker A. Assessing inequalities in preventive care use in Europe. Health Policy. 2013;113:247-57.

43. Sabates $R$, Feinstein $L$. The role of education in the uptake of preventative health care: the case of cervical screening in Britain. Soc Sci Med. 2006;62: 2998-3010.

44. Wardle J, McCaffery K, Nadel M, Atkin W. Socioeconomic differences in cancer screening participation: comparing cognitive and psychosocial explanations. Soc Sci Med. 2004:59:249-61.

45. Stimpson JP, Pagan JA, Chen LW. Reducing racial and ethnic disparities in colorectal cancer screening is likely to require more than access to care. Health Aff. 2012;31:2747-54.

46. Finney Rutten LJ, Nelson DE, Meissner HI. Examination of population-wide trends in barriers to cancer screening from a diffusion of innovation perspective (1987-2000). Prev Med. 2004:38:258-68.

47. Peipins LA, Soman A, Berkowitz Z, White MC. The lack of paid sick leave as a barrier to cancer screening and medical care-seeking: results from the National Health Interview Survey. BMC Public Health. 2012;12:520.

48. Kim S, Kwon S, Subramanina SV. Has the National Cancer Screening Program reduced income inequalities in screening attendance in South Korea? Cancer Causes Control. 2015;26:1617-25.

49. Rakowski W, Meissner H, Vernon SW, Breen N, Rimer B, Clark MA. Correlates of repeat and recent mammography for women ages 45 to 75 in the 2002 to 2003 Health Information National Trends Survey (HINTS 2003). Cancer Epidemiol Biomarkers Prev. 2006;15:2093-101.

50. Rutten LJ, Squiers L, Hesse B. Cancer-related information seeking: hints from the 2003 Health Information National Trends Survey (HINTS). $J$ Health Commun. 2006:11 Suppl 1:147-56

51. Bao Y, Fox SA, Escarce JJ. Socioeconomic and racial/ethnic differences in the discussion of cancer screening: "between-" versus "within-" physician differences. Health Serv Res. 2007;42:950-70. 
52. Tefft N, Kageleiry A. State-level unemployment and the utilization of preventive medical services. Health Ser Res. 2014;49:186-205.

53. Korean Statistical Information Service. Statistics Korea. 2014. http://kosis.kr/. Accessed 21 Dec 2015

54. Pace LE, Keating NL. A systematic assessment of benefits and risks to guide breast cancer screening decisions. JAMA. 2014:311:1327-35.

Submit your next manuscript to BioMed Central and we will help you at every step:

- We accept pre-submission inquiries

- Our selector tool helps you to find the most relevant journal

- We provide round the clock customer support

- Convenient online submission

- Thorough peer review

- Inclusion in PubMed and all major indexing services

- Maximum visibility for your research

Submit your manuscript at www.biomedcentral.com/submit 